\title{
Investigation of the Fluorescence Spectra of Cs-Vapor Layers with Nanometric Thickness
}

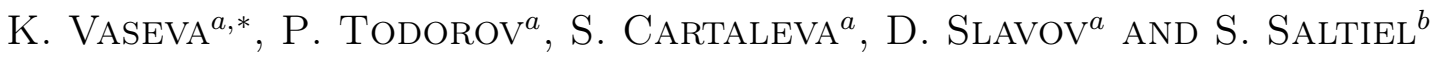 \\ ${ }^{a}$ Institute of Electronics, Bulgarian Academy of Sciences \\ 72 Tzarigradsko Shosse Bld, 1784 Sofia, Bulgaria \\ ${ }^{b}$ Sofia University, Faculty of Physics, 5 J. Bourchier Boulevard, 1164 Sofia, Bulgaria
}

\begin{abstract}
The high resolution spectroscopy of nanometric alkali-vapor layers has been made possible through the development of extremely thin cell. We present a detailed comparison of the fluorescence profiles amplitude and width, obtained in the extremely thin cell, both theoretically and experimentally. Experiments are performed on the $D_{2}$ line of Cs-vapor layer with thickness $L=m \lambda$, where $m=0.5,0.75,1,1.25$. The enhancement rate of the transition profiles width is not growing monotonously, but it is larger for $L$ varying in the interval from $L=0.75 \lambda$ to $L=\lambda$ than that varying in the interval from $L=\lambda$ to $L=1.25 \lambda$. The used theoretical model, based on the optical Bloch equations is in qualitative agreement with the experimental observations.
\end{abstract}

PACS numbers: 32.30.-r, 32.70.Jz

\section{Introduction}

High resolution spectroscopy of atomic layers confined in a thin cell $(10-1000 \mu \mathrm{m})$ is promising for investigation of not only complex spectra of atoms, but also molecules and the properties of light-atom, atom-atom and atom-surface interaction [1]. In the so called extremely thin cells (ETC, of thickness $\leq 1 \mu \mathrm{m}$ ) the sub-Doppler resonances, measured only by a single light beam spectroscopy, are much more pronounced than in thin cells [2]. The vapor-layer thickness can be precisely controlled in an interval around the wavelength of the irradiating laser light $\lambda=852 \mathrm{~nm}$. It was reported that the width of the fluorescence profiles increases monotonously with the cell thickness [3].

Here we present a detailed investigation of experimental and theoretical profiles of the fluorescence on the $D_{2}$ line of Cs-atomic layers with thickness $L=m \lambda$ where $m=0.5,0.75,1,1.25$. We study the fluorescence spectra of the $6 S_{1 / 2}\left(F_{\mathrm{g}}=4\right) \rightarrow 6 P_{3 / 2}\left(F_{\mathrm{e}}=3,4,5\right)$ transitions for different irradiating powers using a frequency tunable single-longitudinal mode diode laser light. Our results show that the width of the fluorescence profiles depends on the atomic vapor layer thickness, and it is growing faster in the interval from $L=0.75 \lambda$ to $L=\lambda$ than in the case of $L=\lambda$ to $L=1.25 \lambda$.

\section{Experimentally and theoretically obtained fluorescence spectra}

In our experiments an extended cavity diode laser (ECDL) is used as radiation source, operating in single-longitudinal mode at $\lambda=852 \mathrm{~nm}$. The emission line

\footnotetext{
* corresponding author; e-mail: kapkavaseva@mail.bg
}

width was measured to be about $3 \mathrm{MHz}$. The laser light is linearly polarized. Its frequency is scanned to match the transitions $6 S_{1 / 2}\left(F_{\mathrm{g}}=4\right) \rightarrow 6 \mathrm{P}_{3 / 2}\left(F_{\mathrm{e}}=3,4,5\right)$ of Cs $D_{2}$ line. The main part of the laser light is sent orthogonally to the ETC windows. The fluorescence in orthogonal to the beam direction is measured by a photodiode. The remaining part of the laser beam is used for laser frequency control. One part of it is sent to a scanning Fabry-Perot interferometer for monitoring the single-mode operation of the ECDL while the second part is sent to a $3 \mathrm{~cm}$ long Cs cell, for registration of saturated absorption (SA) spectrum used as precise frequency reference. The sub-Doppler spectra in the fluorescence are recorded for different cell thickness and laser light intensities.

As the laser beam diameter largely exceeds the cell thickness, the interaction time between an atom and the laser radiation depends on atomic velocity direction. Atoms flying orthogonally to the cell walls have very short interaction time with the light before hitting the cell wall. This time is even shorter than the excited state lifetime and therefore the atoms propagating orthogonally to the cell windows do not have enough time to achieve an absorption-fluorescence cycle. On the other hand, atoms flying parallel to windows benefit significantly longer interaction time before leaving the laser beam and thus mainly contribute to the formation of the fluorescence spectrum. These atoms have zero or very small velocity vector projection on the laser beam direction. The result is narrower signal in the fluorescence than this in the absorption and significantly reduced Doppler broadening.

In Fig. 1, the fluorescence spectra of $6 S_{1 / 2}\left(F_{\mathrm{g}}=4\right) \rightarrow$ $6 P_{3 / 2}\left(F_{\mathrm{e}}=3,4,5\right)$ transitions are presented, for the four different cell thicknesses and high irradiating laser 

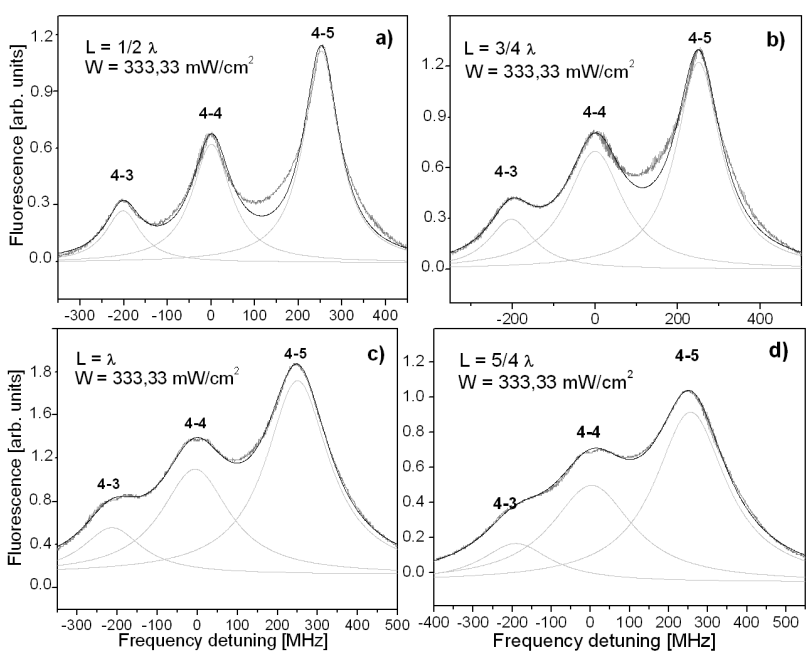

Fig. 1. Fluorescence spectra for $L=0.5 \lambda$ (a), $L=0.75 \lambda(\mathrm{b}), L=\lambda(\mathrm{c}), L=1.25 \lambda(\mathrm{d})$ at high laser power (frequency detuning: from $F_{\mathrm{g}}=4 \rightarrow F_{\mathrm{e}}=4$ transition) fitted with a sum of 3 Lorentz profiles.

power. To compare the width of the fluorescence profiles a fitting procedure is made chasing the width, but not the exact shape of the resonances. We used as a fitting function a sum of 3 Lorentzians, thus excluding the Doppler broadening, which is small in the fluorescence signal of the ETC. From the estimated fit the full width at half maximum (FWHM) and the amplitude of the resonances are deduced. The amplitudes and widths of the fluorescence profiles are increasing with the laser power, for all transitions and all cell thicknesses. The width of the transition profiles is growing with $L$ as well, but differently in each thickness interval.

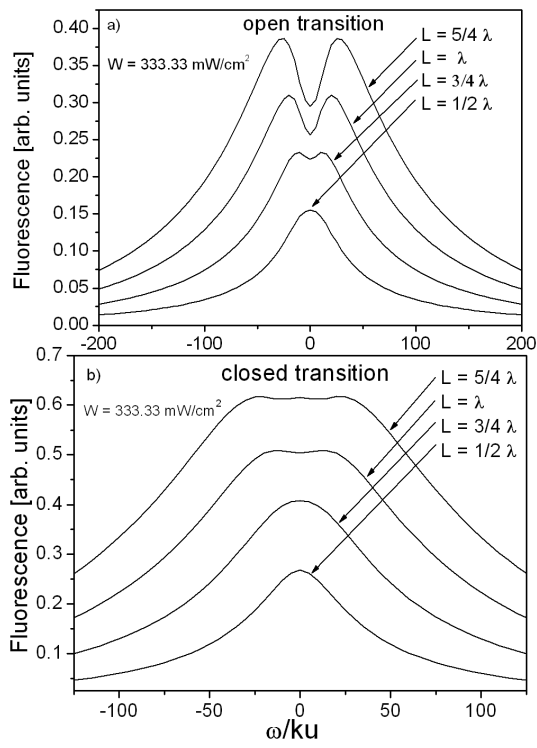

Fig. 2. Theoretical calculations of the fluorescence spectra for open (a) and closed (b) transitions for $L=(0.5,0.75,1,1.25) \lambda$ at high laser power.
We use the developed and described in [4] model, which is based on the optical Bloch equations. The calculations are made for two-level system. Closed or open transitions are considered with parameters applicable to an elementary and realistic case.

For the open transitions (Fig. 2) suffering hyperfine or Zeeman optical pumping, well pronounced narrow dips are observed on the contrary to the case of the closed transitions, where only a small feature is seen for the case of $L=1.25 \lambda$ [5].

\section{Width of the profiles in dependence on the cell thickness}

In Fig. 3, the experimentally and theoretically obtained FWHM of the fluorescence are presented for the $F_{\mathrm{g}}=4 \rightarrow F_{\mathrm{e}}=4$ and $F_{\mathrm{g}}=4 \rightarrow F_{\mathrm{e}}=5$ transitions and for ETC thickness $L=m \lambda$. One can see that the width of the measured and calculated peaks have similar behavior with power density.

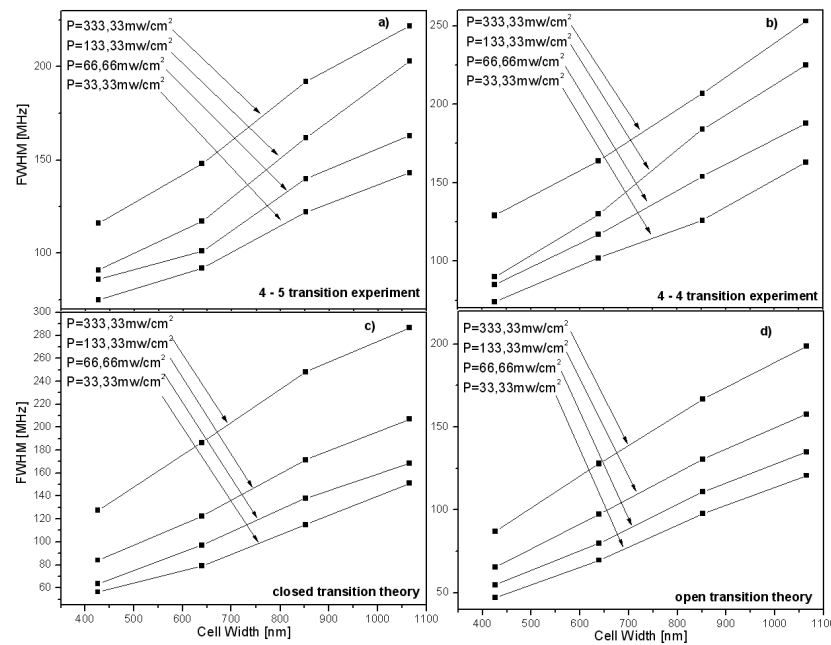

Fig. 3. Fluorescence FWHM for closed transition: experiment (a), theory (c) and for open transition: experiment (b), theory (d) at different light intensities.

With increasing the laser power, the width of the fluorescence profiles increases for all transitions and all cell thicknesses. Also the width of the transition profiles is growing with $L$. However, the rate of the FWHM enhancement is changing. It is smaller for the intervals from $L=0.5 \lambda$ to $L=0.75 \lambda$ and from $L=\lambda$ to $L=1.25 \lambda$ than for the interval from $L=0.75 \lambda$ to $L=\lambda$. The reason for this small difference can be related to a second important process which arises in the ETC - Dicke coherent narrowing (see [4] and references therein). The coherent emission nature of the atomic ensemble leads to relative enhancement of absorption at the optical transition resonance respectively to its wings. The Dicke effect is strongest at $m=0.5$ and exhibits revival for $m=1.5$, 2.5 and collapse for $m=1,2,3$ [6]. Since $m=0.75 \lambda$ and $1.25 \lambda$ are some intermediate cases, the coherent Dicke 
narrowing of absorption still exists for this cell widths. The obtained results can be considered as a support of the assumption that the coherent Dicke narrowing in the ETC absorption can also show some trace in the fluorescence spectra.

\section{Conclusion}

Systematic comparison is made of the fluorescence profile full width at half maximum, estimated theoretically and experimentally. It can be seen that the width and the amplitude of the fluorescence profiles increases with laser power, for all cell thicknesses and all hyperfine transitions. In agreement with the previous results, the width of the transition profiles is growing with the cell thickness. We report here about an additional effect: the enhancement rate of the transition width is not constant, namely it is larger for the vapor layer thickness varying in the interval from $L=0.75 \lambda$ to $L=\lambda$ than that varying in the interval from $L=\lambda$ to $L=1.25 \lambda$.

The presented spectroscopy of the nanometric thickness layer shows that the widths of the fluorescence profiles depend on the layer thickness, even with such small differences as $213 \mathrm{~nm}$. These results can be used for spectral investigations of atoms confined in nanovolumes as well as for spectroscopy of miniature gas discharges.

\section{Acknowledgments}

Authors are grateful for the support by the Bulgarian Fund of Scientific Research (grant: DO02-108/22.05.2009) and Indian-Bulgarian bilateral collaboration (project: BIn-2/07).

\section{References}

[1] S. Briaudeau, D. Bloch, M. Ducloy, Europhys. Lett. 35, 337 (1996).

[2] D. Sarkisyan, D. Bloch, A. Papoyan, M. Ducloy, Opt. Commun. 200, 201 (2001).

[3] D. Sarkisyan, T. Varzhapetyan, A. Sarkisyan, Yu. Malakyan, A. Papoyan, A. Lezama, D. Bloch, M. Ducloy, Phys. Rev. A 69, 065802 (2004).

[4] C. Andreeva, S. Cartaleva, L. Petrov, S.M. Saltiel, D. Sarkisyan, T. Varzhapetyan, D. Bloch, M. Ducloy, Phys. Rev. A 76, 013837 (2007).

[5] P. Todorov, K. Vaseva, S. Cartaleva, D. Slavov, I. Maurin, S. Saltiel, Proc. SPIE 7027, 70270R (2008).

[6] G. Dutier, A. Yarovitski, S. Saltiel, A. Papoyan, D. Sarkisyan, D. Bloch, M. Ducloy, Europhys. Lett. 63, 35 (2003). 